\title{
On the Errors Arising in Surface Integral Equations Due to the Discretization of the Identity Operator ${ }^{\dagger}$
}

\author{
Özgür Ergül ${ }^{1,2}$ and Levent Gürel ${ }^{1,2 *}$ \\ ${ }^{1}$ Department of Electrical and Electronics Engineering \\ ${ }^{2}$ Computational Electromagnetics Research Center (BiLCEM) \\ Bilkent University, TR-06800, Bilkent, Ankara, Turkey \\ \{ergul,lgurel\}@ee.bilkent.edu.tr
}

\section{Introduction}

Surface integral equations (SIEs) are commonly used to formulate scattering and radiation problems involving three-dimensional metallic and homogeneous dielectric objects with arbitrary shapes [1]-[3]. For numerical solutions, equivalent electric and/or magnetic currents defined on surfaces are discretized and expanded in a series of basis functions, such as the Rao-Wilton-Glisson (RWG) functions on planar triangles. Then, the boundary conditions are tested on surfaces via a set of testing functions. Solutions of the resulting dense matrix equations provide the expansion coefficients of the equivalent currents, which can be used to compute the scattered or radiated electromagnetic fields. In general, SIEs involve three basic operators, i.e., integro-differential $\mathcal{K}$ and $\mathcal{T}$ operators, and the identity operator $\mathcal{I}\{\boldsymbol{X}\}(\boldsymbol{r})=\boldsymbol{X}(\boldsymbol{r})$. Depending on the testing scheme and the boundary conditions used, there are four basic SIEs [2],[3], namely, the tangential electric-field integral equation (TEFIE), the normal electric-field integral equation (N-EFIE), the tangential magnetic-field integral equation (T-MFIE), and the normal magnetic-field integral equation (N-MFIE). In the tangential equations, boundary conditions are tested directly by sampling the tangential components of the electric and magnetic fields on the surface. In the normal equations, however, electromagnetic fields are tested after they are projected onto the surface via a cross-product operation with the outward normal vector.

This study consists of two parts. In the first part, we show that the identity operator is truly a major error source in normal and mixed formulations that are discretized with low-order functions, e.g., RWG functions. In the second part, we investigate the incompatibility of SIE formulations in the context of iterative solutions. We show that a compatibility test can be used to determine the breakpoint, where the accuracy of the solution is saturated and cannot be enhanced any more.

\section{Surface Formulations}

Surface formulations are obtained by using one or more SIEs. Using a Galerkin scheme, stable SIE formulations can be categorized into three groups.

- Tangential formulations: T-EFIE for perfectly-conducting objects; the combined tangential formulation (CTF) [3] and the tangential Poggio-Miller-Chang-HarringtonWu-Tsai (T-PMCHWT) [1] formulation for dielectric objects.

- Normal formulations: N-MFIE for perfectly-conducting objects; the combined normal formulation (CNF), the normal Müller formulation (NMF), and the modified normal Müller formulation (MNMF) for dielectric objects [3].

\footnotetext{
${ }^{\dagger}$ This work was supported by the Scientific and Technical Research Council of Turkey (TUBITAK) under Research Grants 105E172 and 107E136, by the Turkish Academy of Sciences in the framework of the Young Scientist Award Program (LG/TUBA-GEBIP/2002-1-12), and by contracts from ASELSAN and SSM.
} 
- Mixed formulations: The combined-field integral equation (CFIE) [2] for both perfectly-conducting and dielectric objects; the electric and magnetic current combined-field integral equation (JMCFIE) for dielectric objects [3].

For a given problem, some of the formulations can be more preferable than the others in terms of efficiency and accuracy.

\section{On the Errors Due to the Identity Operator}

It is well-known that the use of the identity operator in surface formulations affects the conditioning of the resulting matrix equations [3]. Normal and mixed formulations involve well-tested identity operators, which lead to well-conditioned matrix equations that are easy to solve iteratively. Tangential formulations, however, do not contain well-tested identity operators, and their discretizations may lead to ill-conditioned matrix equations. Recent investigations show that the identity operator also plays a key role in the accuracy of the solutions. For example, scattered fields obtained with N-MFIE and CFIE are significantly inaccurate in comparison to those obtained with T-EFIE. Accuracy of N-MFIE and CFIE can be improved to the levels of T-EFIE by increasing the order of discretizations [4]. Similar observations have been made for the solution of dielectric objects [3].

In this paper, we present a computational experiment based on the nonradiating property of the tangential incident fields on an arbitrary surface. Incident fields are expanded in a series of RWG functions by using two methods; an expansion employing tangentially-tested $\mathcal{K}$ and $\mathcal{T}$ operators and an expansion employing well-tested identity operators. We show that the second method using identity operators is significantly inaccurate compared to the first method. The two discretization methods are related to the solutions of electromagnetics problems with SIE formulations, in which the total currents are expanded in a series of basis functions.

Since the excessive discretization error of the identity operator contaminates the accuracy of the normal and mixed formulations, matrix equations obtained with the tangential, normal, and mixed formulations for the same problem are incompatible. For example, a solution vector obtained with CFIE does not satisfy T-EFIE with the desired level of accuracy, and vice versa. Then, in an iterative solution, the minimization of the residual error of a normal or mixed formulation involves a breakpoint, where further reduction of the residual error does not improve the compatibility of the solution with the corresponding tangential formulation. Since the tangential formulations produce more accurate results, the breakpoint also corresponds to the last "useful" iteration, where the accuracy of the solution is saturated and cannot be improved any more. Then, a further reduction of the residual error is practically unnecessary. We conclude that the compatibility of the solution with the corresponding tangential formulation is an important indicator to determine the last useful iteration to obtain the highest possible accuracy with the normal and mixed formulations.

\section{Numerical Examples}

As an example, Figs. 1 and 2 present a set of results involving a sphere of radius $0.5 \lambda$ illuminated by a plane wave:

- The tangential incident fields (nonradiating currents) are expanded in a series of RWG functions on the sphere by using the two methods described above. Expansion coefficients are calculated and used to compute the radiated fields in the far zone. Fig. 1(a) 


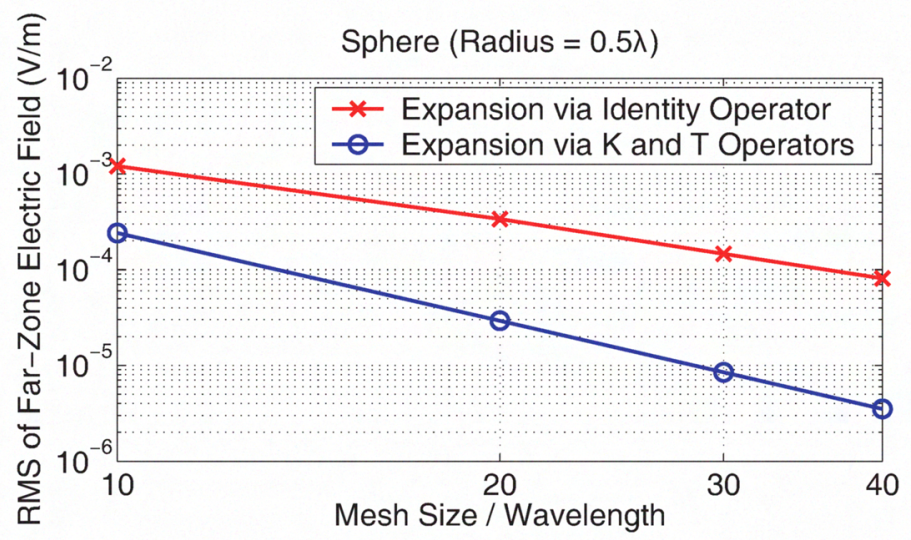

(a)

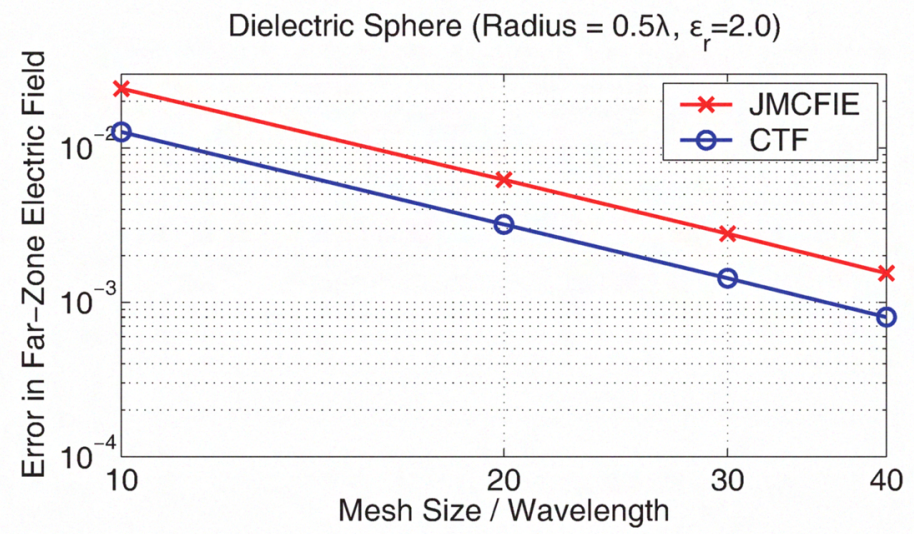

(b)

Fig. 1. Numerical examples to demonstrate the contamination of the accuracy of SIE formulations with the discretization error of the identity operator.

presents the root-mean square (RMS) of the radiated electric field, which should be ideally zero, as a function of the mesh size. We observe that the expansion by using the identity operator is significantly inaccurate compared to the expansion by using the integro-differential operators.

- Fig. 1(b) presents the results of a scattering problem involving a dielectric sphere with a radius of $0.5 \lambda$ and a relative permittivity of 2.0 located in free space. Solutions obtained with CTF and JMCFIE are compared with the analytical results obtained by a Mie-series solution. The normalized error in the far-zone electric field is calculated for various mesh sizes. We observe that the tangential formulation CTF is more accurate than the mixed formulation JMCFIE, which contains well-tested identity operators.

- Fig. 2(a) presents a CFIE $(0.2 \times$ T-EFIE $+0.8 \times \mathrm{N}$-MFIE) solution of a scattering problem involving a perfectly-conducting sphere of radius $0.5 \lambda$. The residual error for CFIE is plotted with respect to BiCGStab iterations. We also plot residual errors "CFIE to EFIE" and "CFIE to MFIE" that are obtained by testing the CFIE solution in the T-EFIE and N-MFIE systems, respectively. The compatibility of the CFIE solution with the T-EFIE and N-MFIE systems is saturated after the 10th iteration. For the same problem, T-EFIE and CFIE solutions are compared in Fig. 2(b). In addition to the residual error, we consider the error in the far-zone electric field (with respect 


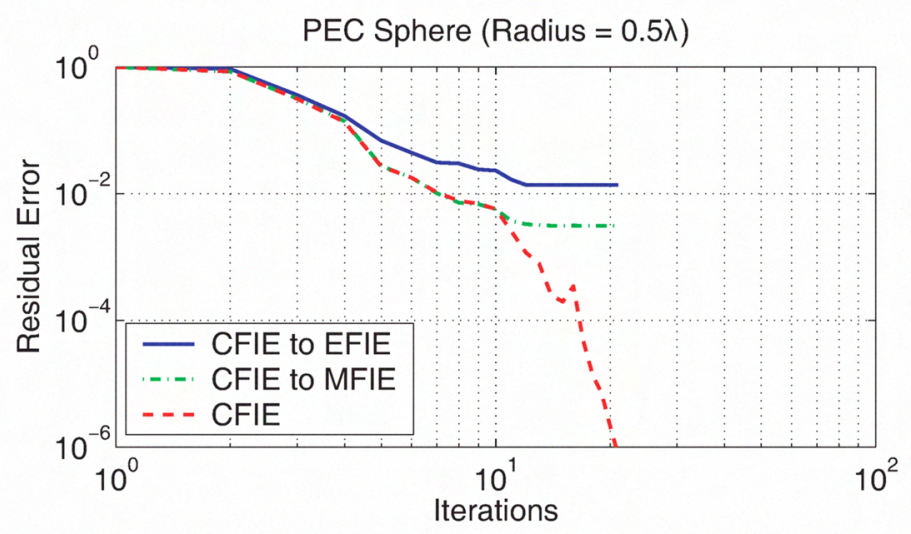

(a)

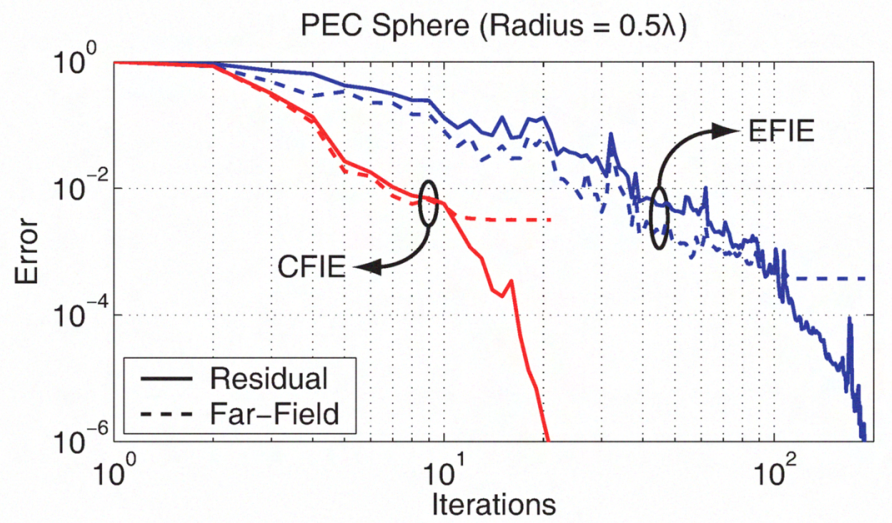

(b)

Fig. 2. Numerical examples to demonstrate the compatibility of SIE formulations in the context of iterative solutions.

to a Mie-series solution), which saturates for both T-EFIE and CFIE. In the case of CFIE, the last useful iteration to obtain the minimum achievable error corresponds to the breakpoint, where the compatibility of the CFIE solution with the T-EFIE system is maximized, i.e., at the 10th iteration.

\section{References}

[1] A. J. Poggio and E. K. Miller, "Integral equation solutions of three-dimensional scattering problems," in Computer Techniques for Electromagnetics, R. Mittra, Ed. Oxford: Pergamon Press, 1973, Chap. 4.

[2] X.-Q. Sheng, J.-M. Jin, J. Song, W. C. Chew, and C.-C. Lu, "Solution of combinedfield integral equation using multilevel fast multipole algorithm for scattering by homogeneous bodies," IEEE Trans. Antennas Propagat., vol. 46, no. 11, pp. 1718-1726, Nov. 1998.

[3] P. Ylä-Oijala, M. Taskinen, and S. Järvenpää, "Surface integral equation formulations for solving electromagnetic scattering problems with iterative methods," Radio Science, vol. 40, RS6002, doi:10.1029/2004RS003169, Nov. 2005.

[4] Ö. Ergül and L. Gürel, "Linear-linear basis functions for MLFMA solutions of magnetic-field and combined-field integral equations," IEEE Trans. Antennas Propagat., vol. 55, no. 4, pp. 1103-1110, Apr. 2007. 